\title{
Review Article \\ Complex Molecules at Liquid Interfaces: Insights from Molecular Simulation
}

\author{
David L. Cheung \\ Department of Pure and Applied Chemistry, University of Strathclyde, Glasgow G1 1XL, UK \\ Correspondence should be addressed to David L. Cheung; david.cheung@strath.ac.uk
}

Received 10 April 2014; Accepted 18 September 2014; Published 28 October 2014

Academic Editor: Fuke Wang

Copyright (C) 2014 David L. Cheung. This is an open access article distributed under the Creative Commons Attribution License, which permits unrestricted use, distribution, and reproduction in any medium, provided the original work is properly cited.

\begin{abstract}
The behaviour of complex molecules, such as nanoparticles, polymers, and proteins, at liquid interfaces is of increasing importance in a number of areas of science and technology. It has long been recognised that solid particles adhere to liquid interfaces, which provides a convenient method for the preparation of nanoparticle structures or to modify interfacial properties. The adhesion of proteins at liquid interfaces is important in many biological processes and in a number of materials applications of biomolecules. While the reduced dimensions of these particles make experimental investigation challenging, molecular simulations provide a natural means for the study of these systems. In this paper I will give an overview of some recent work using molecular simulation to investigate the behaviour of complex molecules at liquid interfaces, focusing on the relationship between interfacial adsorption and molecular structure, and outline some avenues for future research.
\end{abstract}

\section{Introduction}

Dating back to the ancient Greeks the interface between immiscible fluids, in particular between oil and water, has been of both scientific and practical interest [1]. As the two liquid components can have radically different properties, the behaviour of molecules in an interfacial environment can differ from bulk solution. For example, molecular recognition driven by hydrogen bonding can be orders of magnitude stronger at the air-water interface than in solution $[2,3]$, which may be exploited in the formation of ordered materials [4] or the operation of molecular machines at the air-water interface [5]. When additional species are added to the system, these may accumulate at the interface; this is exemplified by amphiphilic surfactants, the adsorption of which to interfaces can be used to modify interfacial properties, such as surface tension. This adsorption of molecules also allows the interfaces to be used as platforms for chemical synthesis [6] and formation of nanoparticles [7]. It has long been recognised that liquid interfaces potentially provide elegant and convenient templates for the construction of dense and ordered structures. While the adsorption and self-assembly of small surfactant molecules have attracted attention over many years, recently the adsorption of larger and more complex molecules, such as nanoparticles, polymers, and proteins, and other biomolecules has attracted attention.

For over 100 years it has been known that solid particles can adhere to interfaces between immiscible liquids [8]. This has been exploited in the formation of two-dimensional colloidal crystals [9] or in the use of colloidal particles to stabilise structures formed through phase separation of immiscible liquids, such as the so-called Pickering emulsions [8] and bijels [10] (particle-stabilised gels). More recently attention has turned to nanometre-sized particles [11, 12]. The adsorption of nanoparticles onto liquid interfaces has been used by a number of groups to create dense nanoparticle monolayers [13] and to create nanoparticle stabilised emulsions (colloidosomes) [14]. Adsorption of metallic or semiconducting nanoparticles at electrified interfaces has the potential to create novel electrooptical devices [15], such as nanoplasmonic sensors or nanoparticle mirrors.

The adsorption of polymers to liquid interfaces has also been used to create highly ordered structures, for both simple and more complex polymer architectures. In particular dendrimers, polymers with a branched, tree-like structure have been shown to self-assemble at liquid interfaces [16], creating highly ordered thin films with applications in organic electronics. The self-assembled structures can also be controlled 
through changes to the solvent phases. In recent work Richmond and coworkers have shown that polyelectrolytes may self-assemble at oil-water interfaces and that the structures formed may be controlled through changes to the $\mathrm{pH}[17$, 18] or the addition of ions [19] to the water subphase. In particular, for polyacrylic acid, desorption from the interface can be triggered by increasing the $\mathrm{pH}$ of the water phase above 4.5 [17], demonstrating the potential for the control of polymer structures at oil-water interfaces.

As well as synthetic molecules, proteins and other biomolecules may adsorb onto liquid interfaces. While in most biological contexts protein adsorption at interfaces is avoided, due to the denaturing effect of the interface, for a number of proteins the interface is their native environment [20] and they fulfil a number of biological functions, including acting as surfactants, catalysis, and immune response. The behaviour of proteins at interfaces is also of interest for many industrial and technological applications. Many foods [21], such as ice cream, mayonnaise, and confectionary products, are emulsions or foams and a large number of proteins are being used as stabilisers for these disperse systems. It has also been shown that oil-water interfaces may be used to prepare highly ordered protein crystals [22].

Despite this intense interest there are still a number of open questions regarding the behaviour of complex molecules, both synthetic and biological, at liquid interfaces. These include the following.

(1) How do complex molecules interact with liquid interfaces?

As the adsorption of molecules, either individually or in larger aggregates, is the initial step in interfacial self-assembly, understanding how these molecules interact with the interface is vital. In addition to giving information regarding the adsorption strength (hence thermodynamic stability), the presence of a barrier to adsorption can lead to reduction in the rate of adsorption of molecules at interfaces.

(2) How do complex molecules assemble at interfaces?

While the formation of dense nanoparticle structures on liquid interfaces has been demonstrated, the controlled formation of more complex structures is still a matter of much investigation. The confinement of molecules to two dimensions can change the structures formed (compared to a fully threedimensional system) and the different properties of the two liquid components can have a strong influence on the interaction between molecules confined to the interface between them. In particular capillary interactions (interactions mediated by the interface) can themselves be affected by pinning of the contact line to the particles [23].

(3) How does adsorption at interfaces affect the dynamics of complex molecules?

Due to the high adsorption strengths at liquid interfaces the motion of adsorbed molecules becomes essentially two-dimensional, leading to transport of properties that can be quite different to bulk solution. This can be particularly complex when the different fluid phases have significantly different viscosities, for instance, when one of the components is a polymer solution or melt.

(4) What conformations do complex molecules adopt at liquid interfaces?

For molecules that possess internal degrees of freedom, such as polymers and proteins, adsorption onto liquid interfaces can alter the molecular conformation. Due to the close relation between their structure and function this is particularly important for proteins, with adsorption on interfaces typically (though not always) associated with a decrease or loss of functionality.

Many of these questions remain unresolved due to the difficulty of studying interfacial systems experimentally; by definition these systems have multiple components, which can be complex to prepare and make separating generic effects from those specific to certain systems difficult, and the properties of interfacial systems can change over length scales as small as Angstroms, which requires a level of spatial resolution that is hard to achieve experimentally. By contrast molecular simulation operates directly on the molecular level, giving a high level of spatial resolution, and simulations can be performed on simple model systems, allowing for the systematic study of effects of, for example, size of colloidal particle or length of polymer chain in a controlled manner.

In this paper I will outline some of my recent work, which has focused using molecular simulation on investigating complex molecules at liquid interfaces, typically focusing on the underlying statistical mechanics of adsorption and on how this can be related to molecular structure and properties. This paper is not designed to be a comprehensive overview of the behaviour of complex molecules at interfaces, rather a short personal account of some recent work and future challenges. The interested reader may find more detailed accounts in a number of excellent review articles (e.g., [2831]).

\section{Nanoparticles}

As outlined in the introduction, it has long been known that solid particles can adhere to liquid interfaces. For colloidal particles $(R \sim \mu m)$ the adsorption strength has been rationalised through changes to the liquid-liquid and particle-liquid surface areas and the adsorption free energy may be written in terms of macroscopic surface tensions as [9]

$$
E=-\gamma_{12} \delta A_{12}+\gamma_{1 P} A_{1 P}+\gamma_{2 P} A_{2 P},
$$

where $\gamma_{i j}$ is the surface tension between components $i$ and $j$ (where $i, j$ are liquid 1 , liquid 2, or colloid/nanoparticle $P), \delta A_{12}$ is the change in surface area between the liquid components, and $A_{i P}$ is the surface area of the particle exposed to liquid component $i$ (illustrated in Figure 1). Using typical values of the surface tension this predicts that the 


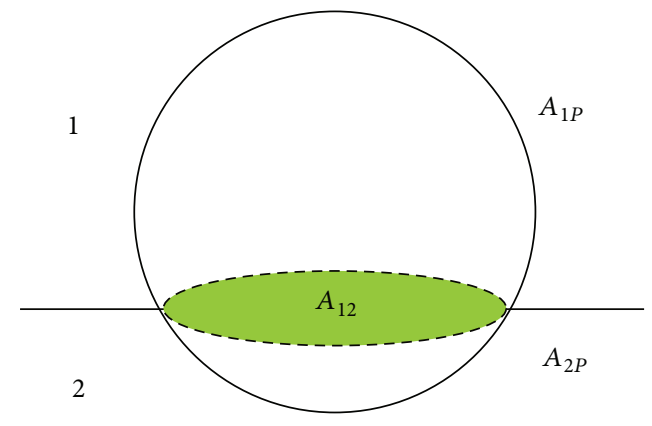

FIGURE 1: Schematic diagram illustrating nanoparticle-solvent contact areas.

adsorption energy of a micron sized colloidal particle is of the order $10^{6} k_{B} T$, explaining the essentially irreversible adsorption of colloidal particles, the formation of colloidal arrays at liquid interfaces, and the using of colloids as emulsion stabilisers (Pickering emulsions). Later, this was generalised by Aveyard and Clint [32] to include the effect of line tension. Negative line tensions were found to increase the particle stability, whereas positive line tension decreased it. For large enough positive line tensions it is possible for this to lead to complete destabilisation of the particle from the interface.

The first attempts to test the applicability of these macroscopic theories to nanoparticles were performed by Bresme and Quirke [33-35]. They found that, under the assumption that particle-liquid surface tensions and line tension depend on particle size, Young's equation

$$
\gamma_{13}-\gamma_{23}-\gamma_{12} \cos \theta+\frac{\tau \cos \theta}{R \sin \theta}=0
$$

could be used to predict the contact angle for nanoparticles at liquid interfaces.

While this tells us about the stability of particles at interfaces, they gave no information on the form of the particle-interface interaction. In some of my recent work, I used molecular simulations to calculate the nanoparticleinterface interaction for a number of simple, model systems. Initially this studied the Widom-Rowlinson mixture [24] (a limiting case of the nonadditive hard sphere mixture):

$$
V_{i j}(r)=\left(1-\delta_{i j}\right) V_{\mathrm{HS}}(r, \sigma),
$$

where $\delta_{i j}$ is the Kronecker delta function and $V_{\mathrm{HS}}(r, \sigma)$ is the hard sphere potential. Monte Carlo simulations, using Wang-Landau sampling [36, 37], were used to calculate the nanoparticle-interface interaction. Comparison with the prediction of Pieranski theory [9] shows that this underestimates both the adsorption strength and the range of the interaction (Figure 2(a)). While it is possible that the underestimation of the barrier is due to the neglect of line tension in Pieranski theory, simply adding this on would not change the interaction range. Rather this underestimation of interaction range arises due to the assumption of a flat, infinitely thin interface in the macroscopic theory (giving an interaction range strictly equal to the particle radius), whereas it is well known that fluctuations in the interface position, from thermal motion (capillary waves) and fluctuations in the bulk density, can lead to an interface that is rough and broad on a microscopic scale. From simulation snapshots it can be seen that these interface fluctuations give an interface width that is comparable in size to the studied nanoparticles and can lead to bridging between the nanoparticle and the interface (Figure 2(b)). Similar results were also by Fan et al. in atomistic simulations of silica nanoparticles at waterdecane interface [38]. In comparison with the simulation results the continuum model also predicts a more rapid variation in the nanoparticle-interface interaction near the interface. It has been shown that inclusion of capillary waves into the continuum models may also lead to a softening in the interaction [23].

In order to test how transferable these results were to other systems more recently I used molecular dynamics simulations (with umbrella sampling [39]) to determine the nanoparticle-interface interaction for a nanoparticle at an interface in a binary Lennard-Jones mixture [25]. As for the Widom-Rowlinson mixture the nanoparticle-interface interaction is both longer ranged and stronger than predicted from macroscopic theories (Figure 3(a)), indicating that this increased interaction range is a general feature of nanoparticles at interfaces. In this case as the particle size and interfacial tension increase, a maximum appears in the nanoparticleinterface interaction, indicating a slight $\left(\sim 2 k_{B} T\right)$ barrier to particle adsorption at the interface. Recent experimental work studying gold nanoparticles that the water-toluene interface has, for certain ligands, also found a similar barrier to interfacial adsorption [40]. It was suggested that the barrier in the experimental system arose due to rearrangement of ligands or to electrostatic interactions. In the simulations, however, these effects are absent and the free energy barrier to adsorption potentially arises due to deformation of the interface, which may be seen from simulation snapshots (Figure 3(b)); for particles at the interface and in bulk solvent, the interface is approximately flat, whereas at intermediate separations the interface noticeably deforms due to the presence of the particles. It should also be noted that, in molecular dynamics simulations of surfactant functionalised nanoparticles by Udayana Ranatunga et al. [41], no barrier to adsorption was seen, indicating that the presence of stabilising ligands is not a sufficient condition for an adsorption barrier.

As well as uniform nanoparticles, particles with anisotropy, in their surface structure and/or shape, have attracted attention. The behaviour of Janus particles, particles with hemispheres of differing functionality, at interface has attracted interest [42]; as they can possess hydrophobic and hydrophilic faces, these can have enhanced stability over uniform particles. Based on macroscopic models, Binks and Fletcher [43] showed that the desorption energy of a Janus particle can be up to three times that of a uniform particle.

Using a modified version of the hard sphere system I investigated the stability of Janus nanoparticles at liquid interfaces [26]. In agreement with expectation, the Janus particles were more stable than uniform particles, with the stability increasing with the surface tension difference $(\Delta \gamma)$ 

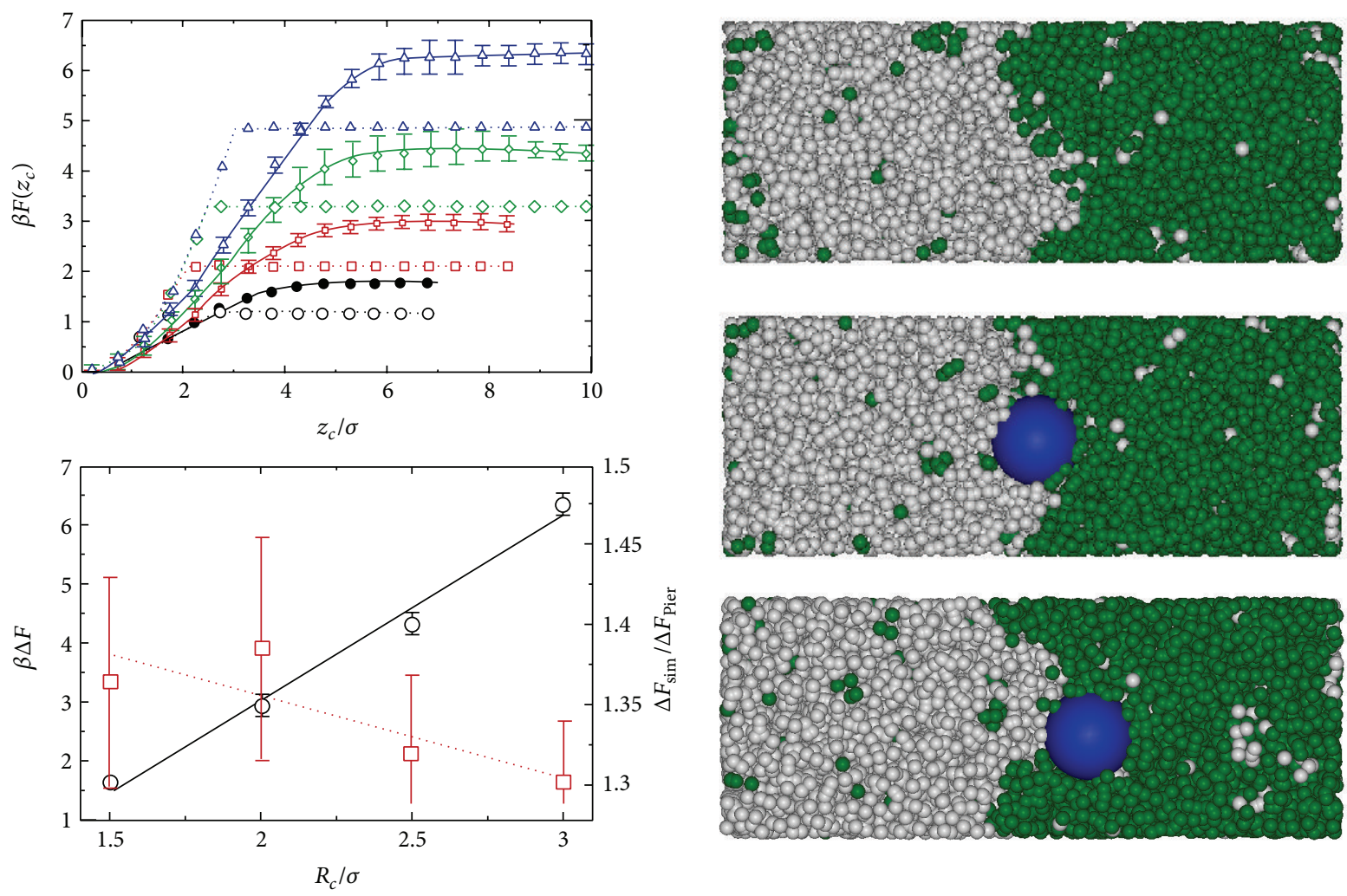

(a)

(b)

Figure 2: (a) (Top) Free energy profile for hard sphere nanoparticle at Widom-Rowlinson interface. Data from nanoparticle radius $R_{c}=$ $1.5 \sigma, 2.0 \sigma, 2.5 \sigma$, and $3.0 \sigma$ denoted by black, red, green, and blue lines, respectively (solid denotes simulation data and dotted line Pieranski approximation). (Bottom) Simulation desorption free energy (black line) and the ratio of the desorption free energies from simulation and Pieranski theory (red). (b) Simulation snapshots showing interface in absence of nanoparticle (top), nanoparticle with $z_{c}=-0.13 \sigma$ (middle), and nanoparticle with $z_{c}=3.7 \sigma$. Figure adapted from [24] $\odot$ American Physical Society.

between the two faces (Figure 4(a)). A similar increase in stability of Janus particles over uniform ones was seen by Fan et al. in the study of silica nanoparticles with varying numbers and locations of hydroxyl groups on the nanoparticle surface [38] and in recent work by Razavi et al. [44]. The stability of the most amphiphilic particle (with faces completely wet by the appropriate solvent), however, was substantially less than three times that of a uniform particle as predicted by Binks and Fletcher. Study of the particle motion showed that the particles possessed a considerable degree of orientational freedom (Figure 4(b)), which was absent in the macroscopic model (in which the particle orientation was assumed to be fixed in the preferred orientation). By performing simulations with the particle orientation fixed in the ideal state, it was found that the particle stability was increased (Figure 4(c)).

As well as the stability of nanoparticles at interfaces, their dynamics and transport properties have attracted interest. The motion of nanoparticles at interfaces is important in understanding their use as tracers in microrheology [45]. Using molecular dynamics simulations, I investigated the diffusion of small nanoparticles at fluid interfaces [46]. Due to the high adsorption energy, the motion of these particles was indeed two-dimensional. Once the two-dimensional confinement of the particles was accounted for, it was found that the particle diffusion was increased relative to bulk solution, which may be understood due to the lower effective viscosity of the interfacial region. The results of this work differed from those of Song et al. [47], studying carbon nanoparticles at a water-PDMS interface. This difference may be due to the larger particles used in that work or the difference in viscosity between the water and polymer components in that work; the diffusion of particles on a fluid interface increases when the viscosity difference between the two fluid components is small [48]. The rotational motion of the particles was also probed and it was found that this differed little from bulk solution; understanding the rotational motion of the nanoparticles at interfaces is important both for their potential application as catalysts and in the synthesis of Janus nanoparticles.

\section{Dendrimers}

Since they were first synthesized, dendrimers [49] have attracted much interest with potential applications in areas including drug delivery [50] and organic electronics [51]. They possess a high degree of symmetry and through a variety of synthetic routes, it is possible to selectively functionalise the dendrimer end groups [52]. This allows the 

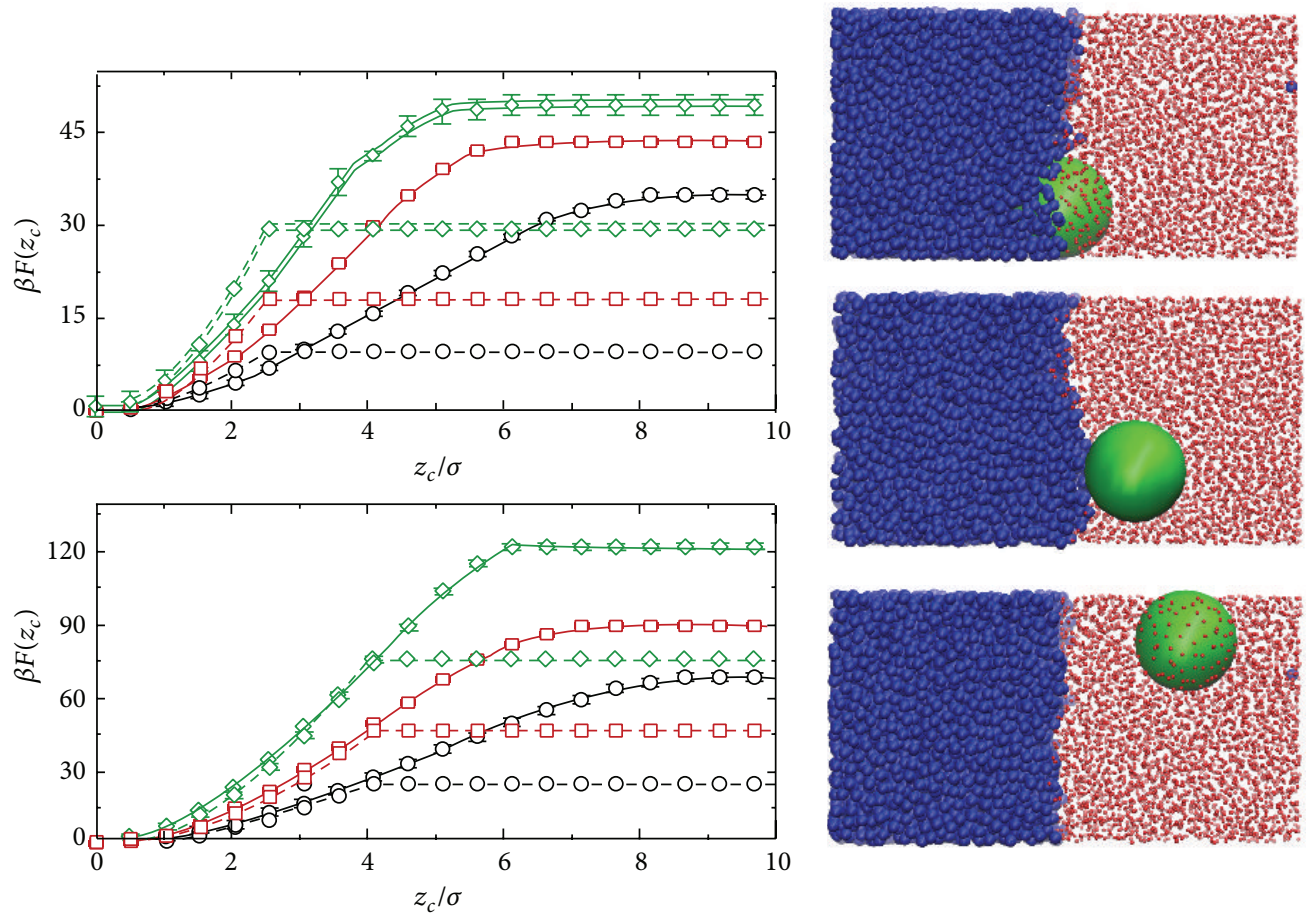

(a)

(b)

Figure 3: (a) Free energy profiles for LJ-nanoparticle with $R_{c}=2.5 \sigma$ (top) and $4.0 \sigma$ (bottom) at liquid-liquid interface. Black, red, and green lines denote solvent density $\rho \sigma^{3}=0.50,0.60$, and 0.69 , respectively. (b) Simulation snapshots showing interface structure for nanoparticle with $z_{c}=0$ (top), $z_{c}=6 \sigma$ (middle), and $z_{c}=9 \sigma$ (bottom). Figure adapted from [25] ® American Institute of Physics.

preparation of amphiphilic or Janus dendrimers, which have been shown to self-assemble into a range of structures in solution [53]. The large number of end groups (even for relatively low generation dendrimers) means that this selective functionalization can have a large effect of the interfacial properties of dendrimers. They have recently been shown to be effective components for interfacial materials, having a range of interesting self-assembly processes [16]. Poly(amino amide) (PAMAM) or poly(propylene imine) dendrimers have attracted particular attention having been shown to form a Langmuir-Blodgett monolayers at the surfaces or interfaces [54].

Using atomistic simulations Nawaz and Carbone studied the behaviour of alkyl-modified PAMAM dendrimers at the air-water interface [55]. In comparison with the dendrimer in bulk water at the interface, it adopts an oblate structure (maximising the decrease in the air-water interfacial area), similar to that predicted for ligand-decorated nanoparticles. The stability of the dendrimer, measured through the number of dendrimer solvent hydrogen bonds, was found to be larger for dendrimers that were fully functionalized with alkyl chains compared to unfunctionalized and semifunctionalized dendrimers. This increase in stability arises due to the high degree of flexibility in these molecules, allowing the hydrophobic end groups to leave the water phase while the hydrophilic core remains in solution.

Following on from this I investigated the adsorption strength of a dendrimer at a model liquid-liquid interface
[27]. On approaching the interface, the dendrimer was found to undergo a similar shape change as the atomistic dendrimers, roughly spherical in bulk, disk-shaped at the interface, and rod-like between these (Figure 5(a)). Even a uniform dendrimer was found to adsorb strongly to the interface, with an adsorption strengths $\sim 100-150 k_{B} T$ (Figure 5(b)). Comparison with nanoparticles showed that this conformational change leads to an increase in stability. Selective functionalization of the dendrimer was found to increase the stability of the dendrimer. As in the atomistic simulations, core-shell dendrimer was more stable at the interface (Figure 5(c)). However, the barrier to enter the favoured solvent for the interior beads was lower than that for the favoured solvent for the terminal beads, demonstrating the flexibility of dendrimer and considerable interaction between the dendrimer interior and the solvent. Simulations of Janus dendrimers (end groups with different solvent affinities) demonstrated that this increased the interfacial adsorption strength, as for Janus nanoparticles [26]; this increase in adsorption strength depended on the placement of the end groups, with dendrimers with these end groups segregated adsorbing most strongly to the interface and dendrimers where these end groups alternate having lower adsorption strengths (dendrimers with randomly distributed end groups have an adsorption strength intermediate between these), demonstrating the importance of the placement of these groups for optimizing the interfacial behaviour of dendrimers (Figure 5(d)). 


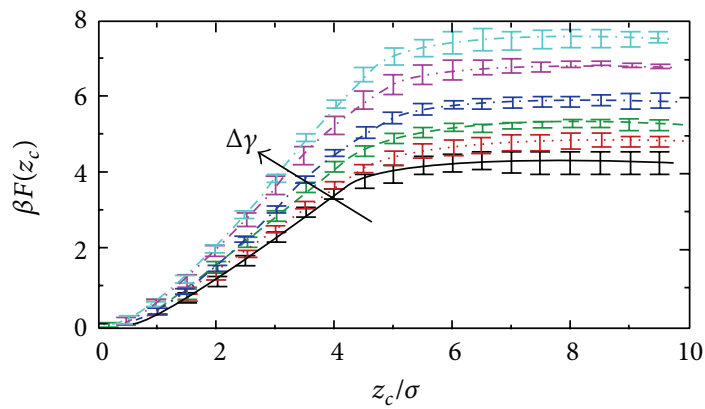

(a)
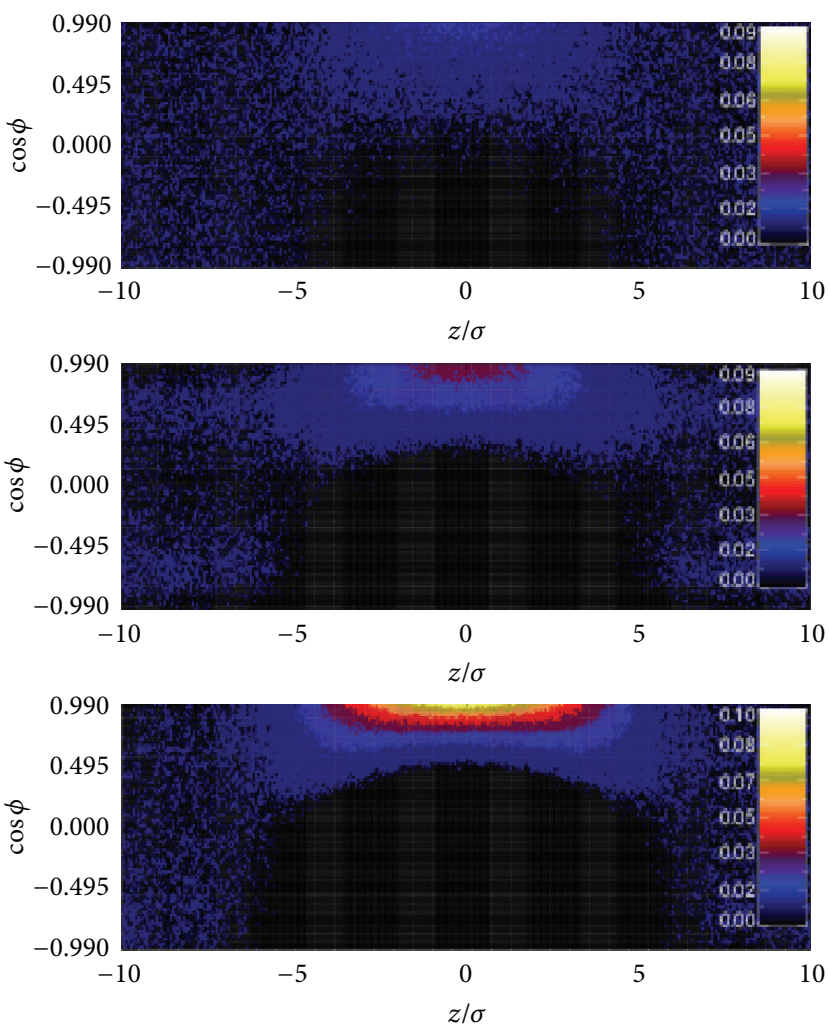

(b)

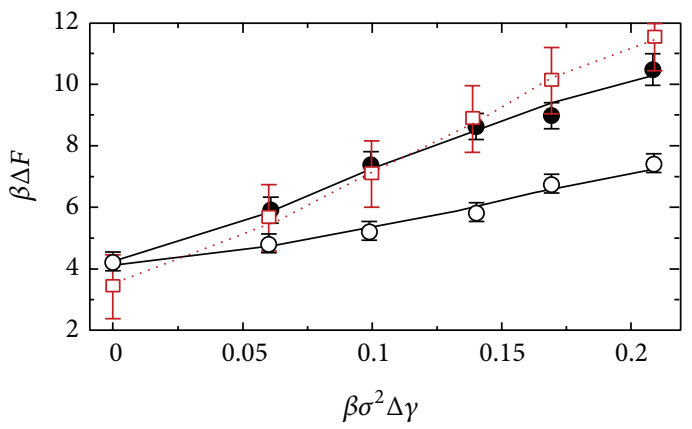

(c)

FIGURE 4: (a) Free energy profiles for Janus nanoparticles at liquid-liquid interface. Data for $\beta \sigma^{2} \Delta \gamma=0,0.06,0.10,0.14,0.17$, and 0.21 denoted by black, red, green, blue, magenta, and turquoise lines, respectively. (b) Joint position-orientation probability distributions for Janus nanoparticles with $\beta \sigma^{2} \Delta \gamma=0.06$ (top), $\beta \sigma^{2} \Delta \gamma=0.10$ (middle), and $\beta \sigma^{2} \Delta \gamma=0.21$ (bottom). (c) Desorption free energy for Janus nanoparticles from simulation (black, circles) and Binks-Fletcher (red, squares). For the simulation data, open symbols denote particles that are free to rotate and filled symbols particles of fixed orientation. Adapted from [26] with permission from The Royal Society of Chemistry.

\section{Proteins}

While proteins may be considered naturally amphiphilic, being composed of a mixture of hydrophobic and hydrophilic amino acids, due to its denaturing effect, adsorption of proteins at interfaces is typically detrimental to their function and in most cases is avoided. For a variety of proteins, however, adsorption at liquid interfaces is an intrinsic part of their function. While these surface-active proteins fulfil a number of functions, including catalysis [56] (lipases) and immune response [57] (lung surfactant proteins), the most common use is as protein surfactants (biosurfactants) [20]. As well as needing to lower surface tension, protein biosurfactants have additional design constraints, such as the desire to minimise aggregation in solution and avoid disruption to the cell membrane. The need to satisfy these somewhat contradictory properties has led to the evolution protein surfactants with a wide range of different structures.

One of the most notable examples of protein surfactants is hydrophobins [58]. These are a class of proteins expressed by certain species of filamentous fungi, which possess a distinctive surface structure. While the majority of the surface 

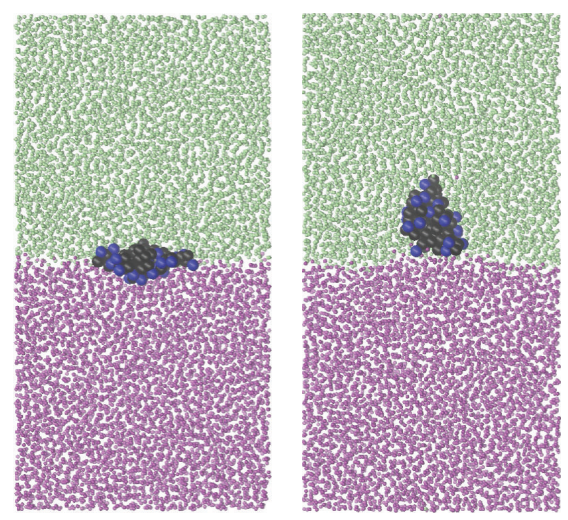

(a)

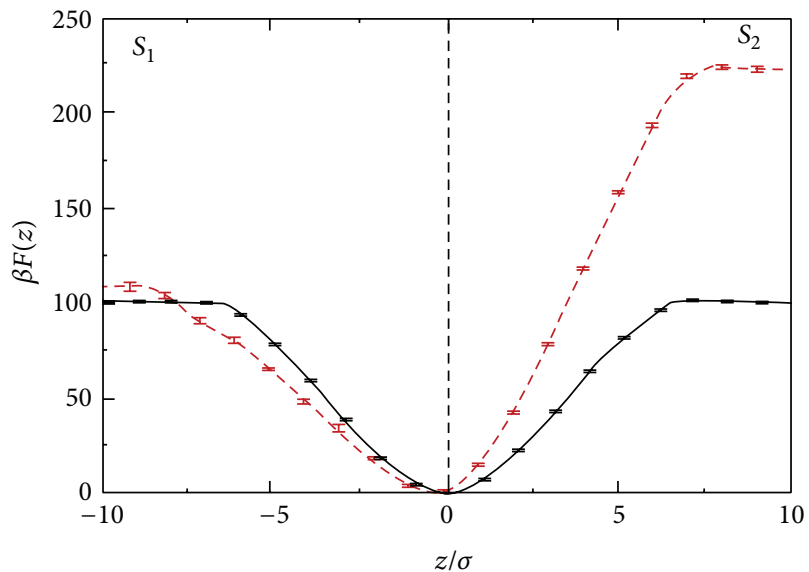

(c)
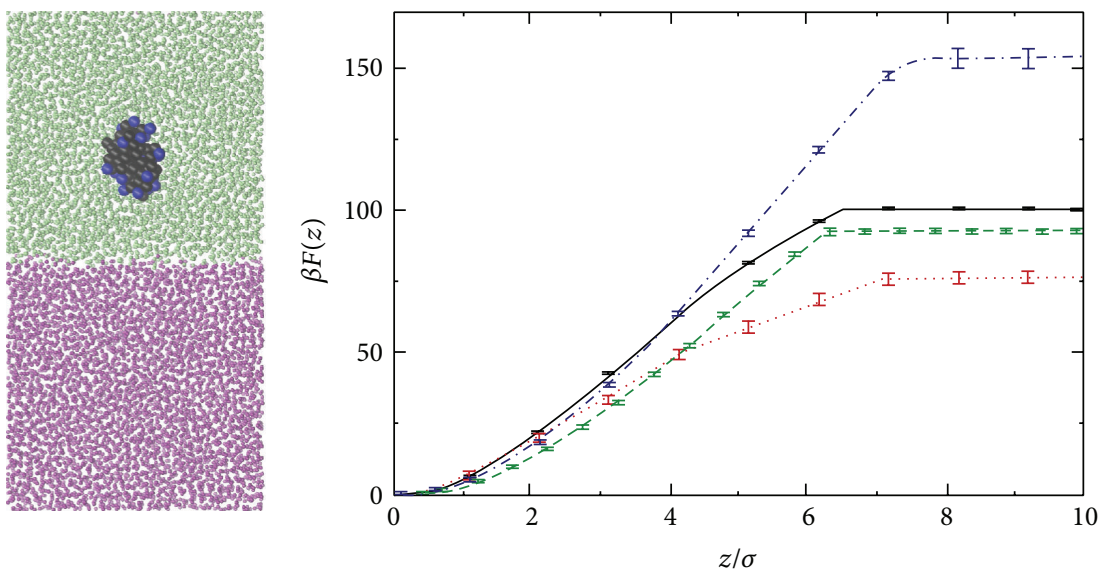

(b)

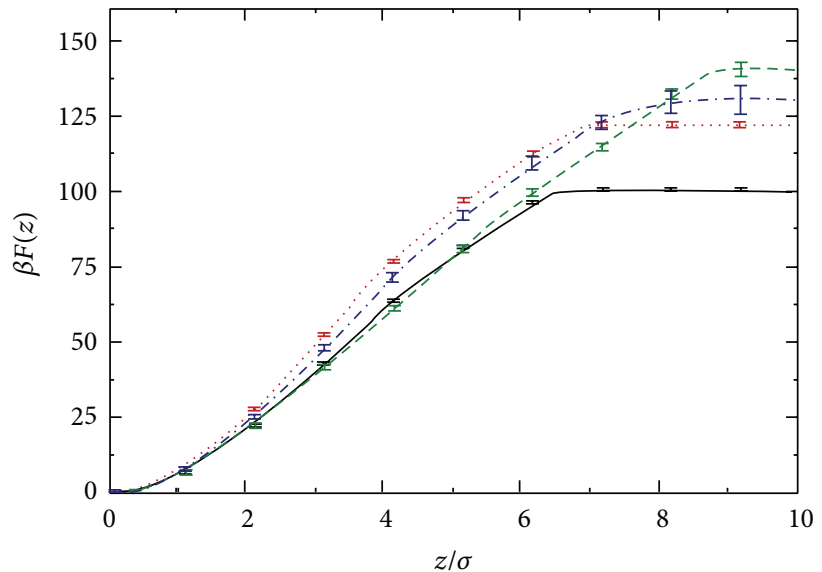

(d)

FIGURE 5: (a) Simulation snapshots showing uniform dendrimer at liquid-liquid interface with nanoparticle-interface separations 0 (left), $6 \sigma$ (middle), and $10 \sigma$ (right). (b) Free energy profile for uniform dendrimers at liquid-liquid interface. Solid line (black) denotes dendrimer in poor solvent, dotted line (red) denotes dendrimer in good solvent, dashed line (green) denotes nanoparticle in poor solvent, and dot-dashed line (blue) denotes nanoparticle in good solvent. (c) Free energy profiles for uniform dendrimer (solid line, black) and core-shell dendrimer (dashed line, red). (d) Free energy profiles for uniform dendrimer (solid line, black), Janus dendrimer with alternating end groups (dotted line, red), Janus dendrimer with segregated end groups (dashed line, green), and Janus dendrimer with randomly distributed end groups (dot-dashed line, blue). Adapted from [27] with permission from The Royal Society of Chemistry.

is hydrophilic, they typically have a patch of hydrophobic residues covering $\sim 20 \%$ of their surface. This amphiphilic structure, essentially making them naturally occurring Janus particles, makes them highly surface active, with them being amongst the most surface active proteins known. Beyond this common surface structure and a well-defined disulphide bond network, the hydrophobins form a diverse group of proteins and are commonly grouped into two classes, differentiated by their solubility and self-assembly at interfaces. As well as fulfilling a number of biological functions, such as formation of aerial structures or mediating fungal adhesion to hydrophobic surfaces, they are being investigated for a number of materials applications, such as foam and emulsion stabilisers $[59,60]$ or biocompatible coatings on drug particles [61]. While such applications, along with their unusual behaviour, have prompted a large number of experimental studies until recent years, simulation studies of hydrophobins have been rarer. Early simulations by Mark et al. examined the structure of SC3, a fibril forming (class-I) hydrophobin in bulk water and at water-hydrophobic interfaces $[62,63]$.

While the adsorption of hydrophobins at oil-water interfaces may be qualitatively understood due to the anisotropic surface structure, it is useful to understand how the adsorption strength is controlled by their sequence and surface structure. Using coarse-grain molecular dynamics simulations, I investigated the adsorption strength of two common type-II hydrophobins (HFBII and HFBI) at the water-octane interface [64]. Unlike class-I hydrophobins, which often undergo significant conformational change at interfaces, class-II hydrophobins have rigid globular structures that remain largely unchanged (Figure 6(a)). Both of these were found to adsorb strongly at the interface with adsorption strengths $\sim 100 k_{B} T$ (comparable to those for nanoparticles), indicating that these essentially adsorb irreversibly onto 

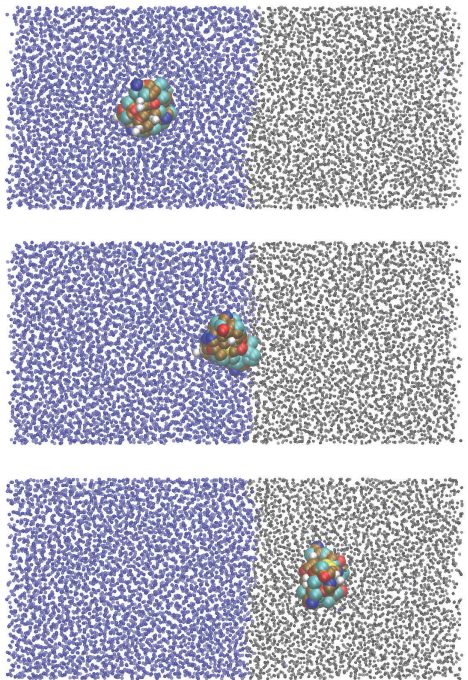

(a)

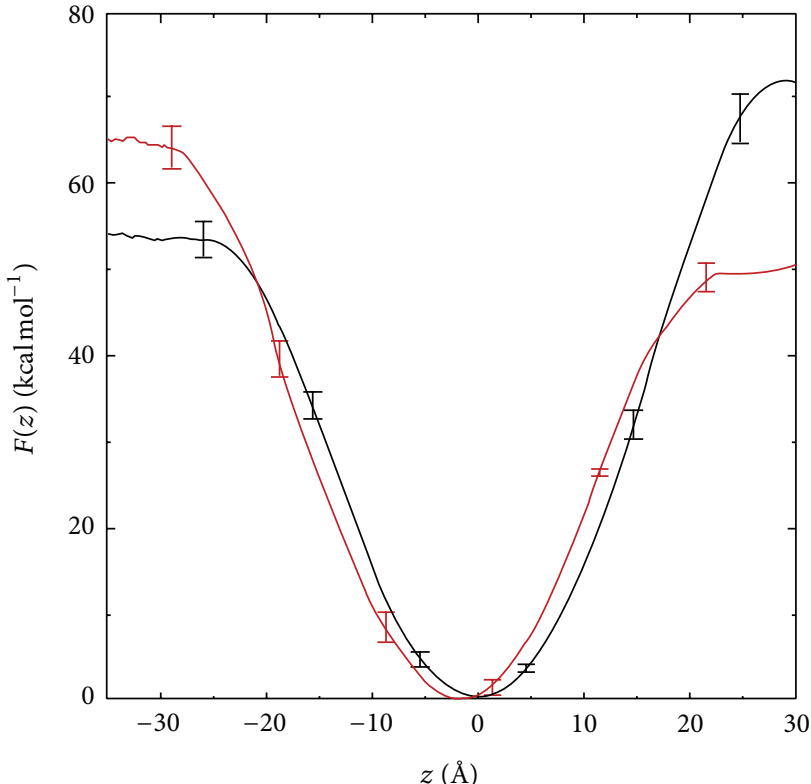

(b)

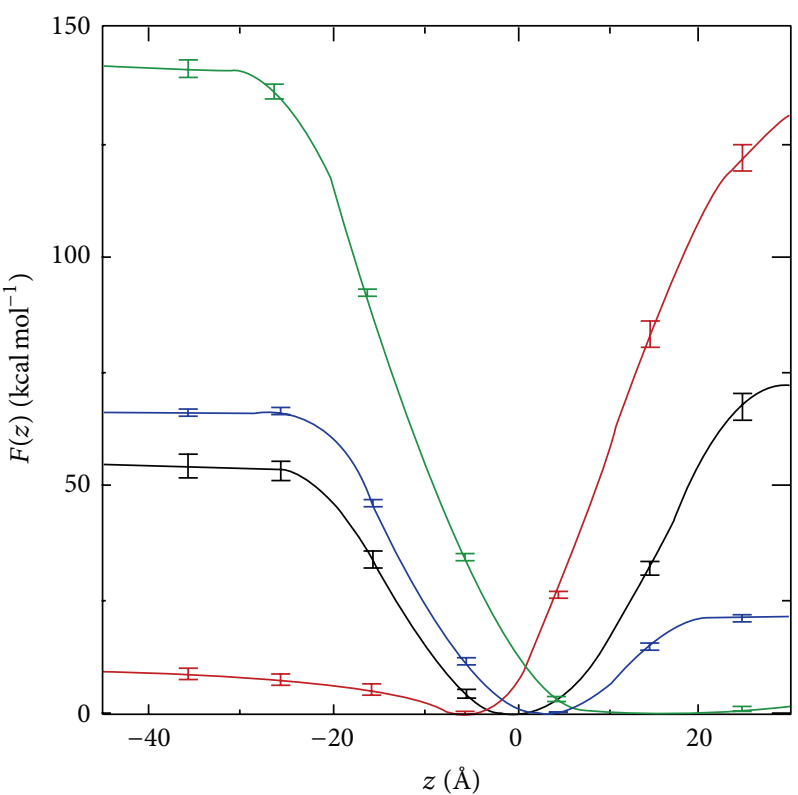

(c)

FIGURE 6: (a) Simulation snapshots showing CG HFBII in bulk water (top), at water-octane interface (middle), and in bulk octane (bottom). (b) Free energy profile for HFBII (black) and HFBI (red). (c) Free energy profiles for native HFBII (black) and hydrophilic (red), hydrophobic (green), and average (blue) HFBII pseudoproteins. Reprinted with permission from D. L. Cheung, Langmuir, 28, 8730 (2012). Copyright 2012 American Chemical Society.

the interface (Figure 6(b)). Comparison between these two showed that HFBII was slightly hydrophilic (the free energy barrier for desorption into the water component was slightly lower than going into octane), whereas HFBI was slightly hydrophobic. While this difference in behaviour is consistent with both the differing hydrophobic surface areas of these proteins and their different biological functions, it is notable that these proteins have both very similar structures and a large degree of sequence similarity. In order to go beyond this simple observational approach, I performed simulations of pseudo-HFBII proteins, where the interactions with the solvent beads were taken to be identical for all the protein beads. Three different cases were considered: the proteinsolvent interactions being uniformly hydrophilic, uniformly hydrophobic, and an average interaction, where the interaction strength between the protein and solvent beads was 
taken to be the average of the interaction strengths for the beads in the native protein (Figure 6(c)). For the first two cases, the pseudo-HFBII was found to be completely destabilised from the interface, with the protein residing in either the water (uniformly hydrophilic) or the octane (uniformly hydrophobic) regions. In contrast, the average pseudo-HFBII was found to be surface active (with a free energy minimum at the interface) but unlike the native protein it is hydrophobic (the barrier to move into the octane region is lower than that for the water region). This can be understood as the hydrophobic patch contains many highly hydrophobic residues that, together with the hydrophobic residues in the core of protein, made the averaged interaction overall hydrophobic (simulations of a single bead from the average protein give an octane-water transfer free energy of $\sim 0.8 \mathrm{kcal} \mathrm{mol}^{-1}$ ). Comparison between the flexible proteins and proteins that are held rigid showed that even in the case of these rigid proteins structural flexibility can still play a significant role in their interfacial adsorption.

The use of a coarse-grain model in this previous work was justified due to the limited conformational change seen in class-II hydrophobins; it is less applicable to classI hydrophobins that often undergo significant structural change at interfaces. As shown by Schulz et al. [65] this change in conformational behaviour can strongly influence the potential application of these proteins, specifically finding that EAS (a class-I hydrophobin) induced the formation of hydroxyapatite at water-hexane interface, whereas HFBII did not, which can be explained by the larger conformational change exhibited by EAS at the interface and in the presence of $\mathrm{Ca}^{2+}$ and $\mathrm{HPO}_{4}{ }^{-}$ions. Simulation of EAS [66] using a multiscale model also showed that its conformational change is responsible for the aggregation of these proteins into fibrils, underlining the importance of accurately representing conformational change in these proteins. Going beyond hydrophobins, Euston has also compared atomistic and coarse-grain models for the simulation of proteins at fluid interfaces [67], finding that CG models gave results similar to atomistic models, although the CG model used underestimated the penetration of the protein (Barley LTP) into the oil phase.

\section{Outlook}

As outlined above over the past few years, much of my work has been aimed at using molecular simulation to examine the behaviour of complex molecules at liquid interfaces, with a particular focus on understanding how the structure and properties of these molecules affect their adsorption strength. Together with work performed by other groups, this has dramatically improved our understanding of the adsorption of nanoparticles at interfaces, in particular on the breakdown of continuum approximations for nanoscale particles, the role of capillary waves, and other microscopic interface deformations on the behaviour of particles, and the effect of nanoparticle surface structure. This has already improved our understanding of the adsorption processes, with current work focusing on understanding the interactions between nanoparticles adsorbed on liquid interfaces and their selfassembly.

While my research to date has largely focused on simple systems, with even the hydrophobins being amongst the simplest possible proteins, there is increasing interest in the behaviour of more complex systems. With improvements to synthetic methodologies it is becoming possible to create nanoparticles [68] and polymers with increasingly complex structures. Notwithstanding recent controversy over stripy nanoparticles $[69,70]$, it is becoming apparent that it is possible to create patterned surfaces with features on the nanometre length scale [71, 72]. These increasingly complex particles will exhibit interesting behaviour at interfaces, with potential applications that extend beyond simple emulsion stabilisers or as templates for the formation of nanostructured materials. For instance Crossley et al. have used structured nanoparticles as phase transfer catalysts for upgrading biofuels [73], with this recently being extended to zeolite nanoparticles [74].

Due to their lack of conformational change at interface, hydrophobins may be regarded as low-hanging fruit compared to other proteins. Understanding conformational change of proteins at oil-water interfaces is a challenging problem for both simulation and experiment. Tensiometry and rheological measurement give indirect insight into changes in the protein conformation [75], neutron/X-ray reflectivity can give average protein sizes and thicknesses of adsorbed layers [76], and circular dichroism and IR spectroscopy can give information about protein secondary structure [77]. Recently the development of synchrotron radiation circular dichroism (SRCD) spectroscopy [78] has allowed the quantitative determine of protein secondary structure at oil-water interfaces. This has been applied to a number of proteins at oil-water interfaces, showing that, in general, there is a tendency for the $\alpha$-helical content of proteins to decrease upon interfacial adsorption [30, 79], although this is, by no means, a universal phenomenon. In contrast to experimental studies simulation may allow the direct visualisation of protein structure at oil-water interfaces. For many years, however, simulations were unable to access timescales necessary to study protein conformations at interfaces. With recent advances in GPU computing [80], CG [81, 82] and hybrid [83] protein models and simulation methods, such as replica exchange [84] or metadynamics [85], will in the coming years make this more feasible. While to date more simulations have focused on short peptides [86] or food proteins [87, 88], these advances in computational power and methodologies will allow for the study of more complex proteins at interfaces, including proteins with complex functionality such as enzymes.

\section{Conflict of Interests}

The author declares that there is no conflict of interests regarding the publication of this paper. 


\section{Acknowledgments}

The research outlined in this paper has benefited from collaboration and helpful conversations with Michael Allen, Stefan Bon, Fernando Bresme, Paola Carbone, Phillip Cox, Russell DeVane, and Martin Oettel. It was supported by UK EPSRC, ERC, and the Leverhulme trust.

\section{References}

[1] C. L. McFearin, D. K. Beaman, F. G. Moore, and G. L. Richmond, "From franklin to today: toward a molecular level understanding of bonding and adsorption at the Oil-Water interface," Journal of Physical Chemistry C, vol. 113, no. 4, pp. 1171-1188, 2009.

[2] X. Cha, K. Ariga, M. Onda, and T. Kunitake, "Molecular recognition of aqueous dipeptides by noncovalently aligned oligoglycine units at the air/water interface," Journal of the American Chemical Society, vol. 117, no. 48, pp. 11833-11838, 1995.

[3] K. Ariga, T. Nakanishi, J. P. Hill et al., “Tunable pK of amino acid residues at the air-water interface gives an L-zyme (Langmuir Enzyme)," Journal of the American Chemical Society, vol. 127, no. 34, pp. 12074-12080, 2005.

[4] K. Ariga, M. V. Lee, T. Mori, X.-Y. Yu, and J. P. Hill, "Twodimensional nanoarchitectonics based on self-assembly," Advances in Colloid and Interface Science, vol. 154, no. 1-2, pp. 20-29, 2010.

[5] K. Ariga, T. Mori, and J. P. Hill, "Evolution of molecular machines: from solution to soft matter interface," Soft Matter, vol. 8, no. 1, pp. 15-20, 2012.

[6] A. Fallah-Araghi, K. Meguellati, J. Baret et al., "Enhanced chemical synthesis at soft interfaces: a universal reaction-adsorption mechanism in microcompartments," Physical Review Letters, vol. 112, no. 2, Article ID 028301, 2014.

[7] A. I. Campbell, R. A. Dryfe, and M. D. Haw, "Deposition and aggregation of au at the liquid/liquid interface," Analytical Sciences, vol. 25, no. 2, pp. 307-310, 2009.

[8] S. U. Pickering, "CXCVI.--emulsions," Journal of the Chemical Society, Transactions, vol. 91, pp. 2001-2021, 1907.

[9] P. Pieranski, "Two-dimensional interfacial colloidal crystals," Physical Review Letters, vol. 45, p. 569, 1980.

[10] P. S. Clegg, "Fluid-bicontinuous gels stabilized by interfacial colloids: low and high molecular weight fluids," Journal of Physics: Condensed Matter, vol. 20, no. 11, Article ID 113101, 2008.

[11] Y. Lin, H. Skaff, T. Emrick, A. D. Dinsmore, and T. P. Russell, "Nanoparticle assembly and transport at liquid-liquid interfaces," Science, vol. 299, no. 5604, pp. 226-229, 2003.

[12] Y. Lin, A. Böker, H. Skaff et al., "Nanoparticle assembly at fluid interfaces: structure and dynamics," Langmuir, vol. 21, no. 1, pp. 191-194, 2005.

[13] A. Böker, J. He, T. Emrick, and T. P. Russell, "Self-assembly of nanoparticles at interfaces," Soft Matter, vol. 3, no. 10, pp. 12311248, 2007.

[14] A. D. Dinsmore, M. F. Hsu, M. G. Nikolaides, M. Marquez, A. R. Bausch, and D. A. Weitz, "Colloidosomes: selectively permeable capsules composed of colloidal particles," Science, vol. 298, no. 5595, pp. 1006-1009, 2002.

[15] M. E. Flatté, A. A. Kornyshev, and M. Urbakh, "Nanoparticles at electrified liquid-liquid interfaces: new options for electrooptics," Faraday Discussions, vol. 143, pp. 109-115, 2009.
[16] M. J. Felipe, N. Estillore, R. B. Pernites, T. Nguyen, R. Ponnapati, and R. C. Advincula, "Interfacial behavior of OEGlinear dendron monolayers: aggregation, nanostructuring, and electropolymerizability," Langmuir, vol. 27, no. 15, pp. 93279336, 2011.

[17] D. K. Beaman, E. J. Robertson, and G. L. Richmond, "Unique assembly of charged polymers at the oil-water interface," Langmuir, vol. 27, no. 6, pp. 2104-2106, 2011.

[18] E. J. Robertson and G. L. Richmond, "Chunks of charge: effects at play in the assembly of macromolecules at fluid surfaces," Langmuir, vol. 29, no. 35, pp. 10980-10989, 2013.

[19] D. K. Beaman, E. J. Robertson, and G. L. Richmond, "Metal ions: driving the orderly assembly of polyelectrolytes at a hydrophobic surface," Langmuir, vol. 28, no. 40, pp. 1424514253, 2012.

[20] A. Cooper and M. W. Kennedy, "Biofoams and natural protein surfactants," Biophysical Chemistry, vol. 151, no. 3, pp. 96-104, 2010.

[21] J. Ubbink, "Soft matter approaches to structured foods: from "cook-and-look" to rational food design?" Faraday Discussions, vol. 158, pp. 9-35, 2012.

[22] B. R. Silver, V. Fülöp, and P. R. Unwin, "Protein crystallization at oil/water interfaces," New Journal of Chemistry, vol. 35, no. 3, pp. 602-606, 2011.

[23] H. Lehle and M. Oettel, "Stability and interactions of nanocolloids at fluid interfaces: effects of capillary waves and line tensions," Journal of Physics: Condensed Matter, vol. 20, no. 40, Article ID 404224, 2008.

[24] D. Cheung and S. Bon, "Interaction of nanoparticles with ideal liquid-liquid interfaces," Physical Review Letters, vol. 102, Article ID 066103, 2009.

[25] D. L. Cheung, "Molecular dynamics study of nanoparticle stability at liquid interfaces: effect of nanoparticle-solvent interaction and capillary waves," The Journal of Chemical Physics, vol. 135, Article ID 054704, 2011.

[26] D. L. Cheung and S. A. F. Bon, "Stability of Janus nanoparticles at fluid interfaces," Soft Matter, vol. 5, no. 20, pp. 3969-3976, 2009.

[27] D. L. Cheung and P. Carbone, "How stable are amphiphilic dendrimers at the liquid-liquid interface?" Soft Matter, vol. 9, no. 29, pp. 6841-6850, 2013.

[28] F. Bresme and M. Oettel, "Nanoparticles at fluid interfaces," Journal of Physics Condensed Matter, vol. 19, no. 41, Article ID 413101, 2007.

[29] M. E. Flatté, A. A. Kornyshev, and M. Urbakh, “Understanding voltage-induced localization of nanoparticles at a liquid-liquid interface," Journal of Physics Condensed Matter, vol. 20, no. 7, Article ID 073102, 2008.

[30] J. L. Zhai, L. Day, M.-I. Aguilar, and T. J. Wooster, "Protein folding at emulsion oil/water interfaces," Current Opinion in Colloid and Interface Science, vol. 18, no. 4, pp. 257-271, 2013.

[31] S. Razavi, J. Koplik, and I. Kretzschmar, "Molecular dynamics simulations: insight into molecular phenomena at interfaces," Langmuir, vol. 30, no. 38, pp. 11272-11283, 2014.

[32] R. Aveyard and J. H. Clint, "Particle wettability and line tension," Journal of the Chemical Society-Faraday Transactions, vol. 92, no. 1, pp. 85-89, 1996.

[33] F. Bresme and N. Quirke, "Computer simulation study of the wetting behavior and line tensions of nanometer size particulates at a liquid-vapor interface," Physical Review Letters, vol. 80, no. 17, pp. 3791-3794, 1998. 
[34] F. Bresme and N. Quirke, "Computer simulation of wetting and drying of spherical particulates at a liquid-vapor interface," Journal of Chemical Physics, vol. 110, no. 7, pp. 3536-3547, 1999.

[35] F. Bresme and N. Quirke, "Nanoparticulates at liquid/liquid interfaces," Physical Chemistry Chemical Physics, vol. 1, pp. 21492155, 1999.

[36] F. Wang and D. P. Landau, "Efficient, multiple-range random walk algorithm to calculate the density of states," Physical Review Letters, vol. 86, no. 10, pp. 2050-2053, 2001.

[37] F. Calvo, "Sampling along reaction coordinates with the WangLandau method," Molecular Physics, vol. 100, no. 21, pp. 34213427, 2002.

[38] H. Fan, D. E. Resasco, and A. Striolo, "Amphiphilic silica nanoparticles at the decane-water interface: insights from atomistic simulations," Langmuir, vol. 27, no. 9, pp. 5264-5274, 2011.

[39] G. M. Torrie and J. P. Valleau, "Nonphysical sampling distributions in Monte Carlo free-energy estimation: umbrella sampling," Journal of Computational Physics, vol. 23, no. 2, pp. 187-199, 1977.

[40] K. Du, E. Glogowski, T. Emrick, T. P. Russell, and A. D. Dinsmore, "Adsorption energy of nano- and microparticles at liquidliquid interfaces," Langmuir, vol. 26, no. 15, pp. 12518-12522, 2010.

[41] R. J. K. Udayana Ranatunga, R. J. B. Kalescky, C.-C. Chiu, and S. O. Nielsen, "Molecular dynamics simulations of surfactant functionalized nanoparticles in the vicinity of an oil/water interface," Journal of Physical Chemistry C, vol. 114, no. 28, pp. 12151-12157, 2010.

[42] N. Glaser, D. J. Adams, A. Böker, and G. Krausch, "Janus particles at liquid-liquid interfaces," Langmuir, vol. 22, no. 12, pp. 5227-5229, 2006.

[43] B. P. Binks and P. D. I. Fletcher, "Particles adsorbed at the oilwater interface: a theoretical comparison between spheres of uniform wettability and “Janus" particles," Langmuir, vol. 17, no. 16, pp. 4708-4710, 2001.

[44] S. Razavi, J. Koplik, and I. Kretzschmar, "The effect of capillary bridging on the Janus particle stability at the interface of two immiscible liquids," Soft Matter, vol. 9, no. 18, pp. 4585-4589, 2013.

[45] J. Wu and L. L. Dai, "One-particle microrheology at liquidliquid interfaces," Applied Physics Letters, vol. 89, Article ID 094107, 2006.

[46] D. L. Cheung, "Molecular simulation of nanoparticle diffusion at fluid interfaces," Chemical Physics Letters, vol. 495, no. 1-3, pp. 55-59, 2010.

[47] Y. Song, M. Luo, and L. L. Dai, "Understanding nanoparticle diffusion and exploring interfacial nanorheology using molecular dynamics simulations," Langmuir, vol. 26, no. 1, pp. 5-9, 2010.

[48] M. Negishi, T. Sakaue, and K. Yoshikawa, "Mismatch of bulk viscosity reduces interfacial diffusivity at an aqueous-oil system," Physical Review E, vol. 81, no. 2, Article ID 020901, 2010.

[49] D. A. Tomalia and J. M. Fréchet, "Introduction to 'dendrimers and dendritic polymers," Progress in Polymer Science, vol. 30, no. 3-4, pp. 217-219, 2005.

[50] E. R. Gillies and J. M. J. Fréchet, "Dendrimers and dendritic polymers in drug delivery," Drug Discovery Today, vol. 10, no. 1, pp. 35-43, 2005.

[51] J. Li and D. Liu, "Dendrimers for organic light-emitting diodes," Journal of Materials Chemistry, vol. 19, pp. 7584-7591, 2009.

[52] B. M. Rosen, C. J. Wilson, D. A. Wilson, M. Peterca, M. R. Imam, and V. Percec, "Dendron-mediated self-assembly, disassembly, and self-organization of complex systems," Chemical Reviews, vol. 109, no. 11, pp. 6275-6540, 2009.

[53] V. Percec, D. A. Wilson, P. Leowanawat et al., "Self-assembly of janus dendrimers into uniform dendrimersomes and other complex architectures," Science, vol. 328, no. 5981, pp. 1009-1014, 2010.

[54] K. W. Chooi, A. I. Gray, L. Tetley, Y. Fan, and I. F. Uchegbu, "The molecular shape of poly(propylenimine) dendrimer amphiphiles has a profound effect on their self assembly," Langmuir, vol. 26, no. 4, pp. 2301-2316, 2010.

[55] S. Nawaz and P. Carbone, "Stability of amphiphilic dendrimers at the water/air interface," Journal of Physical Chemistry B, vol. 115, no. 42, pp. 12019-12027, 2011.

[56] P. Reis, K. Holmberg, H. Watzke, M. E. Leser, and R. Miller, "Lipases at interfaces: a review," Advances in Colloid and Interface Science C, vol. 147-148, pp. 237-250, 2009.

[57] J. R. Wright, "Immunomodulatory functions of surfactant," Physiological Reviews, vol. 77, no. 4, pp. 931-962, 1997.

[58] M. B. Linder, "Hydrophobins: proteins that self assemble at interfaces," Current Opinion in Colloid \& Interface Science, vol. 14, no. 5, pp. 356-363, 2009.

[59] F. L. Tchuenbou-Magaia, I. T. Norton, and P. W. Cox, "Hydrophobins stabilised air-filled emulsions for the food industry," Food Hydrocolloids, vol. 23, no. 7, pp. 1877-1885, 2009.

[60] A. R. Cox, D. L. Aldred, and A. B. Russell, "Exceptional stability of food foams using class II hydrophobin HFBII," Food Hydrocolloids, vol. 23, no. 2, pp. 366-376, 2009.

[61] H. K. Valo, P. H. Laaksonen, L. J. Peltonen, M. B. Linder, J. T. Hirvonen, and T. J. Laaksonen, "Multifunctional hydrophobin: toward functional coatings for drug nanoparticles," ACS Nano, vol. 4, no. 3, pp. 1750-1758, 2010.

[62] R. Zangi, M. L. De Vocht, G. T. Robillard, and A. E. Mark, "Molecular dynamics study of the folding of hydrophobin SC3 at a hydrophilic/hydrophobic interface," Biophysical Journal, vol. 83, no. 1, pp. 112-124, 2002.

[63] H. Fan, X. Wang, J. Zhu, G. T. Robillard, and A. E. Mark, "Molecular dynamics simulations of the hydrophobin SC3 at a hydrophobic/hydrophilic interface," Proteins: Structure, Function and Genetics, vol. 64, no. 4, pp. 863-873, 2006.

[64] D. L. Cheung, "Molecular simulation of hydrophobin adsorption at an oil-water interface," Langmuir, vol. 28, no. 23, pp. 8730-8736, 2012.

[65] A. Schulz, M. Fioroni, M. B. Linder et al., "Exploring the mineralization of hydrophobins at a liquid interface," Soft Matter, vol. 8, no. 44, pp. 11343-11352, 2012.

[66] A. de Simone, C. Kitchen, A. H. Kwan, M. Sunde, C. M. Dobson, and D. Frenkel, "Intrinsic disorder modulates protein selfassembly and aggregation," Proceedings of the National Academy of Sciences of the United States of America, vol. 109, no. 18, pp. 6951-6956, 2012.

[67] S. R. Euston, "Molecular dynamics simulation of protein adsorption at fluid interfaces: a comparison of all-atom and coarse-grained models," Biomacromolecules, vol. 11, no. 10, pp. 2781-2787, 2010.

[68] S. C. Glotzer and M. J. Solomon, "Anisotropy of building blocks and their assembly into complex structures," Nature Materials, vol. 6, no. 8, pp. 557-562, 2007.

[69] Y. Cesbron, C. P. Shaw, J. P. Birchall, P. Free, and R. Lévy, "Stripy nanoparticles revisited," Small, vol. 8, no. 24, pp. 3714-3726, 2012. 
[70] M. Yu and F. Stellacci, "Response to "stripy nanoparticles revisited”," Small, vol. 8, no. 24, pp. 3720-3726, 2012.

[71] C. P. Shaw, D. G. Fernig, and R. Lévy, "Gold nanoparticles as advanced building blocks for nanoscale self-assembled systems," Journal of Materials Chemistry, vol. 21, no. 33, pp. 1218112187, 2011.

[72] C. Singh, P. K. Ghorai, M. A. Horsch et al., "Entropy-mediated patterning of surfactant-coated nanoparticles and surfaces," Physical Review Letters, vol. 99, no. 22, Article ID 226106, 2007.

[73] S. Crossley, J. Faria, M. Shen, and D. E. Resasco, "Solid nanoparticles that catalyze biofuel upgrade reactions at the water/oil interface," Science, vol. 327, no. 5961, pp. 68-72, 2010.

[74] P. A. Zapata, J. Faria, M. P. Ruiz, R. E. Jentoft, and D. E. Resasco, "Hydrophobic zeolites for biofuel upgrading reactions at the liquid-liquid interface in water/oil emulsions," Journal of the American Chemical Society, vol. 134, no. 20, pp. 8570-8578, 2012.

[75] B. S. Murray, "Interfacial rheology of food emulsifiers and proteins," Current Opinion in Colloid \& Interface Science, vol. 7, pp. 426-431, 2002.

[76] X. Zhao, F. Pan, and J. R. Lu, "Interfacial assembly of proteins and peptides: recent examples studied by neutron reflection," Journal of the Royal Society Interface, vol. 6, no. 5, pp. S659-S670, 2009.

[77] J. T. Pelton and L. R. McLean, "Spectroscopic methods for analysis of protein secondary structure," Analytical Biochemistry, vol. 277, no. 2, pp. 167-176, 2000.

[78] L. Day, J. Zhai, M. Xu, N. C. Jones, S. V. Hoffmann, and T. J. Wooster, "Conformational changes of globular proteins adsorbed at oil-in-water emulsion interfaces examined by synchrotron radiation circular dichroism," Food Hydrocolloids, vol. 34, pp. 78-87, 2014.

[79] J. Zhai, A. J. Miles, L. K. Pattenden et al., "Changes in $\beta$ lactoglobulin conformation at the oil/water interface of emulsions studied by synchrotron radiation circular dichroism spectroscopy," Biomacromolecules, vol. 11, no. 8, pp. 2136-2142, 2010.

[80] J. A. Anderson, C. D. Lorenz, and A. Travesset, "General purpose molecular dynamics simulations fully implemented on graphics processing units," Journal of Computational Physics, vol. 227, no. 10, pp. 5342-5359, 2008.

[81] L. Monticelli, S. K. Kandasamy, X. Periole, R. G. Larson, D. P. Tieleman, and S.-J. Marrink, "The MARTINI coarse-grained force field: extension to proteins," Journal of Chemical Theory and Computation, vol. 4, no. 5, pp. 819-834, 2008.

[82] R. Devane, W. Shinoda, P. B. Moore, and M. L. Klein, "Transferable coarse grain nonbonded interaction model for amino acids," Journal of Chemical Theory and Computation, vol. 5, no. 8, pp. 2115-2124, 2009.

[83] W. Han, C.-K. Wan, F. Jiang, and Y.-D. Wu, "PACE force field for protein simulations. 1. full parameterization of version 1 and verification," Journal of Chemical Theory and Computation, vol. 6, no. 11, pp. 3373-3389, 2010.

[84] D. J. Earl and M. W. Deem, "Parallel tempering: theory, applications, and new perspectives," Physical Chemistry Chemical Physics, vol. 7, pp. 3910-3916, 2005.

[85] A. Laio and F. L. Gervasio, "Metadynamics: a method to simulate rare events and reconstruct the free energy in biophysics, chemistry and material science," Reports on Progress in Physics, vol. 71, no. 12, Article ID 126601, 2008.

[86] V. Knecht, " $\beta$-Hairpin folding by a model amyloid peptide in solution and at an interface," The Journal of Physical Chemistry B, vol. 112, no. 31, pp. 9476-9483, 2008.
[87] S. R. Euston, P. Hughes, M. A. Naser, and R. E. Westacott, “Comparison of the adsorbed conformation of barley lipid transfer protein at the decane-Water and vacuum-Water interface: a molecular dynamics simulation," Biomacromolecules, vol. 9, no. 5, pp. 1443-1453, 2008.

[88] S. R. Euston, U. Bellstedt, K. Schillbach, and P. S. Hughes, "The adsorption and competitive adsorption of bile salts and whey protein at the oil-water interface," Soft Matter, vol. 7, no. 19, pp. 8942-8951, 2011. 

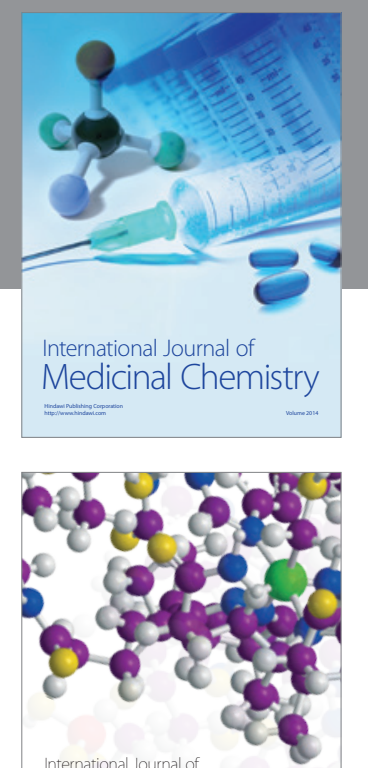

\section{Carbohydrate} Chemistry

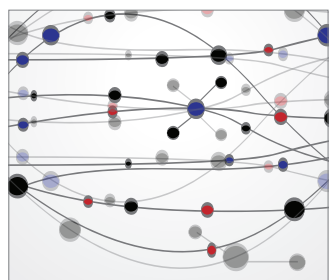

The Scientific World Journal
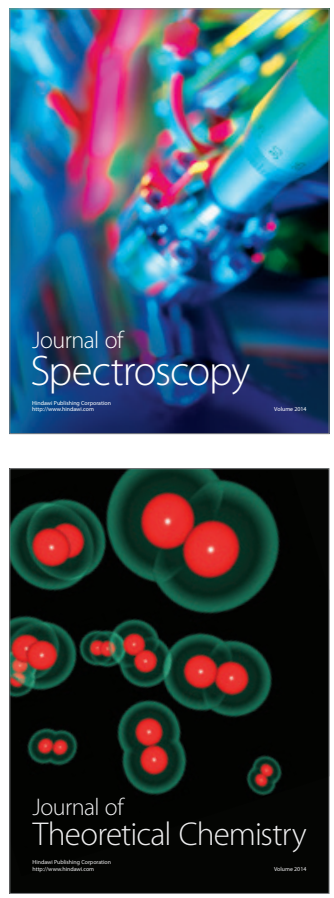
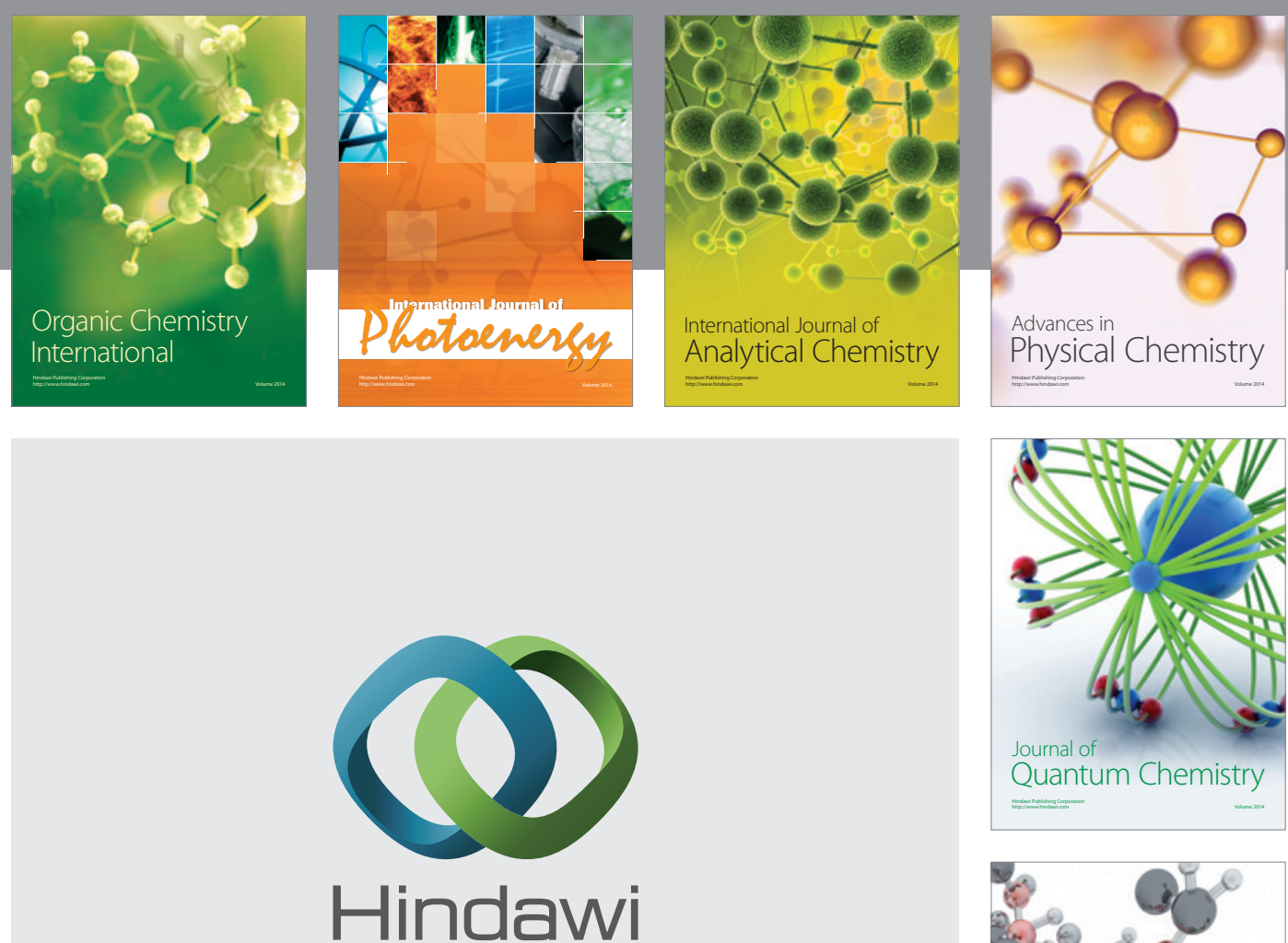

Submit your manuscripts at

http://www.hindawi.com

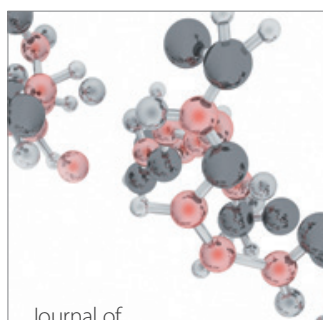

Analytical Methods

in Chemistry

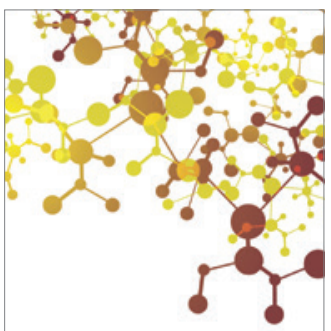

Journal of

Applied Chemistry

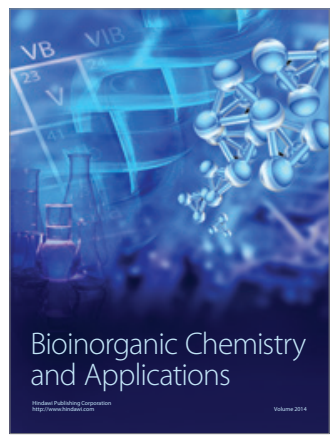

Inorganic Chemistry
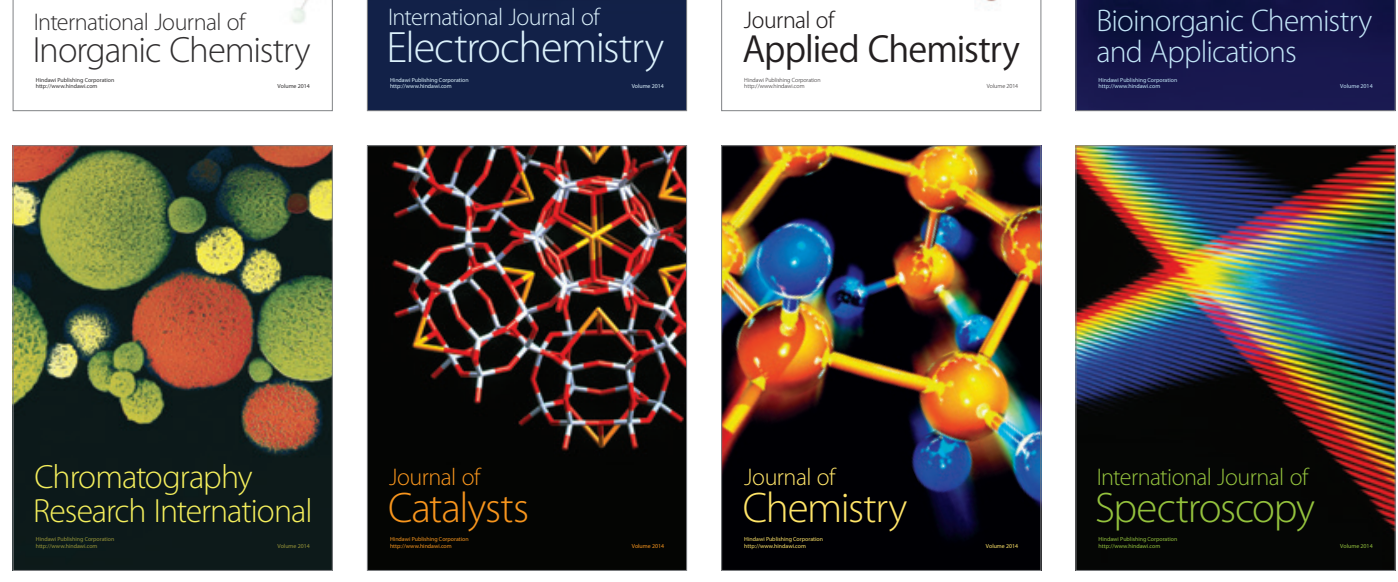